\title{
The electromagnetic calorimeter of ATLAS and its performances
}

\author{
S. Jézéquel ${ }^{1}$
}

ATLAS Collaboration

\begin{abstract}
After a period of testbeam in the beginning of the 90's, the ATLAS collaboration has begun to build a liquid argon/lead electromagnetic calorimeter. Its engineering is a compromise between technical feasibility and optimization of the response to electrons and photons in the LHC environment (especially the channel $\mathrm{H} \rightarrow \gamma \gamma$ ). Special care is taken for a good uniformity over the whole detector. We will describe the final design of the electromagnetic calorimeter with its expected performances.
\end{abstract}

Talk presented at Frontier Detector for Frontier Physics, $7^{\text {th }}$ Pisa Meeting on Advanced Detectors,May 1997 


\section{Introduction}

The ATLAS detector is designed to cover the physics of LHC (Higgs, SUSY, $B$ and top physics). It is composed of an inner tracker inside a solenoidal magnetic field, calorimeters and muon chambers inside a toroidal magnetic field. The electromagnetic calorimeters are designed to identify and measure the direction and the energy of electrons and photons. They are based on liquid argon calorimetry with electrodes and lead absorbers having an accordion shape. They have been designed using the knowledge acquired with the prototypes tested by the RD3 collaboration [1].

\section{The calorimeters of ATLAS}

The calorimetry in ATLAS is divided in two parts. A tile calorimeter lays at radius $>2518 \mathrm{~mm}$ and covers up to $\eta=1$.7. It is made of iron sampled by scintillators. A tube of liquid argon [3] lays inside covering up to $\eta=4.9$. The electromagnetic calorimeters have lead absorbers with accordion shape (barrel) and spanish fan shape (endcap). Each endcap cryostat contains an electromagnetic endcap, a hadronic one and a forward calorimeter. The hadronic calorimeter is made of copper plates. The electromagnetic (hadronic) part of the forward is made of copper (tungsten).

The design of the electromagnetic calorimeter was constrained by the $H \rightarrow \gamma \gamma$ $\left(80 \mathrm{GeV}<m_{H}<130 \mathrm{GeV}\right)$ and $H \rightarrow Z Z^{(*)} \rightarrow e^{+} e^{-} e^{+} e^{-}(120 \mathrm{GeV}<$ $\left.m_{H}<500 \mathrm{GeV}\right)$. The ATLAS collaboration wants to reach the following performances :

- an energy resolution with a sampling term of $10 \%$ and a constant term of $0.7 \%$ up to $5 \mathrm{TeV}$

- a resolution in the measurement of the direction of photons close to $\sigma=$ $\frac{50 \mathrm{mrad}}{\sqrt{E}}$

- an ability to reduce the $\pi^{\circ}$ contribution (produced in jet fragmentation and already selected as isolated from jets) by a factor 3 keeping $90 \%$ of the isolated photons

\section{Principles of Liquid Argon calorimetry at LHC}

The liquid calorimetry is interesting thanks to its uniform response over a large volume. The argon has the property of being resistant to ionizing and neutron radiations. 
To have a calorimeter which has $24 X_{o}$ depth with a reasonable size, particles have to cross successively lead (absorber) and liquid argon. The gap between two absorber plates is $4 \mathrm{~mm}$. The electrodes are positioned in the middle of the gaps; they are held at high voltage while the absorbers are at ground potential in order to create an electrical field $(10 \mathrm{kV} / \mathrm{cm})$ in the argon. The electrons ionized from argon atoms are collected by the electrodes. The RD3 collaboration has proposed to arrange the absorbers and electrodes with an accordion shape. The signal is transmitted from the electrode surface to the front or back face of the calorimeter without having cracks in $\eta$ and $\phi$. In order to cope with the high frequency of interactions at LHC (40 MHz), the electric current extracted from the calorimeter (400 ns long) is transformed by a bipolar shaper in order to measure its amplitude after $40 \mathrm{~ns}$.

\section{The elements of the calorimeters}

The barrel electromagnetic calorimeter is divided in two half parts placed in a cryostat. Each one contains 1024 absorbers and electrodes superposed in the transverse plane. The absorbers have a different lead thickness in $\eta$ to have a constant sampling term over $\eta$ but the sum of lead and epoxy glue thicknesses is constant. The sum of inactive material in front of the calorimeter (inner tracker, inner wall of the cryostat, solenoid, non instrumented liquid argon) is $1.8 X_{o}$ at $\eta=0$. A presampler [2] made of electrodes laying in liquid argon (2 $\mathrm{cm}$ depth), samples the electromagnetic shower just after the cryostat wall. It measures the energy lost in front of the active part of the calorimeter.

The two endcaps are divided in two concentric wheels. The absorbers (756 for the outer wheel and 258 for the inner one) have a spanish fan shape and a constant lead thickness. To minimize the effects of the sampling fraction and argon gap variations with radius, the high voltage which defines the drift velocity is varied. A presampler is put in the shadow zone of the barrel to sample the shower. To allow access to the electronics of the endcaps and the barrel, the readout is outside the cryostat.

The electrodes are made of three copper circuits: the two outers carry high voltage and the inner is connected to the readout. Two layers of polyimide decouple the high voltage and signal layers. The epoxy glue which is used to bind the layers is radiation hard as polyimide. To have a uniform material with respect to thermal expansion, we have chosen to use Kapton $E$ (Dupont de Nemours) which behaves like copper. Since the copper is closer to the absorber thermal expansion coefficient than standard polyimide, the global mechanical uniformity is enhanced too. To minimize the cracks in $\eta$ between electrodes, large size electrodes $\left(2 \times 1 \mathrm{~m}^{2}\right)$ have been developed with industries. The barrel is divided in two electrodes with their common border at $\eta=0.8$ (Fig. 1). Each 
wheel of the endcap contains one type of electrode.

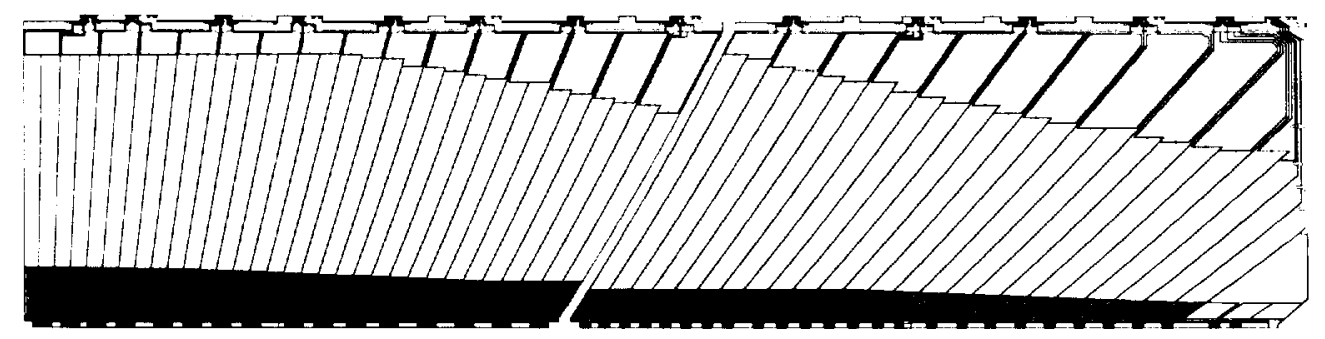

Fig. 1. Design of the barrel electrodes

The cells of the three samplings in depth, are directly drawn on the copper layers of the electrodes. The front sampling is highly granular to disentangle a single photon from a pair of photons created by a $\pi^{o}\left(P_{T}=50 \mathrm{GeV}\right)$. It is thin in depth ( $6 X_{o}$ including all the material in front of the active part of the calorimeter) and granular to measure precisely the impact point. Since the $\eta$ direction is crucial for photons, it is granular in $\eta$. The middle sampling measures the impact point in the $\eta$ and $\phi$ direction. Combining the measurement with the one of the front sampling and the vertex constraint in $\phi$, we are able to measure the photon direction. To contain showers from photons of $P_{T}=50$ $\mathrm{GeV}$ and to minimize the contribution of the electronic noise, this sampling stops at $24 X_{o}$ (except at low $\eta$ ). The last sampling completes the detection zone and allows to measure precisely the energy of electrons of $300 \mathrm{GeV}$ and above. The combination of the three samplings gives a tool to differentiate electrons and $\pi^{ \pm}$. At $\eta>2.5$, there is no front sampling: photons from $\pi^{o}$ are too close compared to the lateral width of each shower and the contribution from pile-up is too large.

\section{Performances}

Figure 2 presents the energy resolution at high luminosity estimated from simulation. It is dominated by the sampling term if the constant term is kept below $1 \%$. At $\eta=1.5$, the energy resolution is degraded because of the transition between the barrel and endcap calorimeters. Figure 3 shows the direction resolution which reaches the ATLAS goal. The isolated $\pi^{\circ}$ with $P_{T}=50 \mathrm{GeV}$ (main reducible background to the $H \rightarrow \gamma \gamma$ search) are rejected by, at least, a factor 3 with a $90 \%$ efficiency for isolated photons. The rejection factor is 2.5 for isolated $\pi^{\circ}$ with $P_{T}=80 \mathrm{GeV}$.

At low luminosity, the channel $H \rightarrow Z Z^{(*)} \rightarrow 4$ leptons is interesting. The main background comes from non resonant $\mathrm{ZZ}$ (irreducible) and $t \bar{t}$ (reducible). The reducible background is removed by asking at least a pair of leptons having 


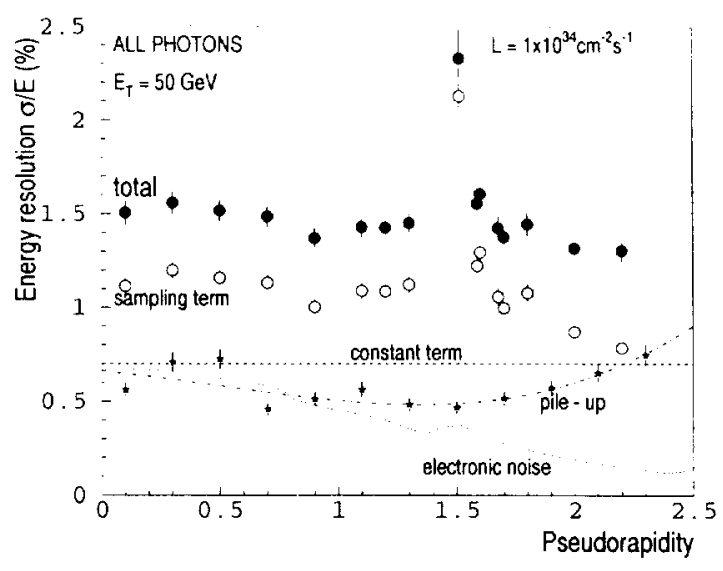

Fig. 2. Energy resolution

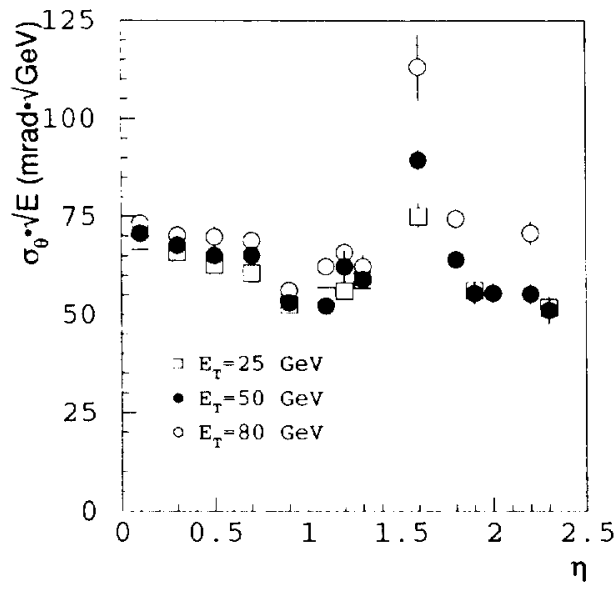

Fig. 3. Angular resolution

a mass close to $\mathrm{Z}$ and a common vertex to the four isolated leptons. The mass resolution relies on the direction measurement with the tracker and the energy measurement of the calorimeter. We will reach a mass resolution of $1.5 \mathrm{GeV}$. The mass range ( $120 \mathrm{GeV}$ to $500 \mathrm{GeV}$ ) will be covered with an integrated luminosity of $3.10^{4} \mathrm{pb}^{-1}$.

The channel $H \rightarrow \gamma \gamma$ is used to search Higgs for masses below $120 \mathrm{GeV}$ at high luminosity. The characteristics of the photons (energy and direction) can only be measured by the calorimeter. We can reach a mass resolution of 1.3 $\mathrm{GeV}\left(\mathrm{m}_{\mathrm{H}}=100 \mathrm{GeV}\right)$ dominated by the energy resolution. The background comes from irreducible $\gamma \gamma$ and reducible $\gamma$-jet. A jet producing an isolated $\pi^{o}$ of $50 \mathrm{GeV}$ can simulate a $\gamma$ in the calorimeter. Using the highly granular front sampling, we can diminish the reducible background in order to get a ratio Signal $/ \sqrt{\text { background }}=3.9$ with an integrated luminosity of $10^{5} \mathrm{pb}^{-1}$.

\section{Conclusion}

According to simulations which are able to reproduce the measurements obtained by RD3, the ATLAS electromagnetic calorimeter fulfill the requirements of LHC. It has the potential to discover the Higgs particle at low and high luminosity. The construction of the test modules will be completed this year. The whole calorimeter has to be finished and tested by 2003 to begin data taking in the year 2005 . 


\section{References}

[1] B. Aubert et al. (RD3 Collaboration), Nucl. Instr. and Meth. A 309 (1992) 438; A 321 (1992) 467; A 325 (1993) 118.

[2] R.A. Davis et al. (RD3 Collaboration) Nucl. Instr. and Meth. A 385 (1997) 47.

[3] CERN/LHCC/96-40;96-41 